\title{
La integración eléctrica en el Perú: Implicancias y retos*
}

\author{
Fiorella García Carthy \\ Abogada por la Pontificia Universidad Católica del Perú. \\ Diplomada en International Commercial Arbitration por American University - \\ Washington College of Law. \\ Edgar Zevallos Salazar \\ Abogado por la Universidad Nacional de San Agustín. \\ Maestría en Derecho de la Empresa por la Universidad Peruana de Ciencias Aplicadas.
}

SUMARIO:

I. Introducción.

II. Marco Teórico.

1. ¿Qué es la Integración Eléctrica?

2. Experiencias internacionales de éxito en Integración Eléctrica.

2.1. Europa.

2.2. América del Norte.

2.3. América Latina.

3. Ventajas y desventajas en la experiencia internacional.

iii. Panorama Actual en el Perú.

1. Interconexión Perú - Ecuador.

2. Interconexión Perú - Brasil.

3. Interconexión Perú - Chile.

4. Interconexión Perú - Bolivia.

5. Consideraciones sobre la integración eléctrica regional.

IV. Conclusiones y Propuestas.

NOTA RECTIFICATORIA: Mediante la presente nota la Asociación Civil ADV Editores aclara que el presente artículo fue incluido dentro de la Edición $N^{\circ} 35$ de ADVOCATUS, cuya fecha de publicación fue el 10 de junio del 2018, de la página 203 hasta la página 214. La Asociación Civil ADV Editores expresa su profundo compromiso con sus estándares de calidad en los productos que ofrece, por lo tanto, vuelve a publicar el presente artículo incluyendo el correcto citado y referencia a las fuentes utilizadas por ambos autores. La Asociación Civil ADV Editores lamenta cualquier afectación que se haya generado por un error involuntario no atribuible a la Asociación. 


\section{RESUMEN:}

Con integración eléctrica se hace referencia al proceso en el cual un conjunto determinado de países decide, a través de un pacto expreso, interconectarse a través de sus sistemas eléctricos. El presente artículo detalla los avances de este proceso, analizando las distintas experiencias internacionales, presentando un panorama actual del Perú y ofreciendo interesantes propuestas y conclusiones al respecto.

Palabras Clave: Integración eléctrica, Interconexión de sistemas electrónicos, Política energética internacional, Transacciones internacionales, Integración eléctrica regional.

\section{ABSTRACT:}

The concept of electrical integration refers to the process by which a group of countries decide to interconnect via electrical systems by means of an express agreement. The present article details the progress of said process and analyzes different international experiences, presenting the current panorama in Peru, offering interesting proposals and conclusions.

Keywords: Electrical integration, Interconnection of electrical systems, International Energy Policy, International Transactions, Regional Electric Integration.

\section{INTRODUCCIÓN}

Vivimos en un país privilegiado, pues gracias a nuestra geografía y diversidad de recursos naturales, el Perú cuenta con un inmenso potencial para la generación de energía, a través de fuentes hidroeléctricas, explotadas solo en un $20 \%$ de su potencial; fuentes térmicas, sobre todo a gas natural; así como fuentes renovables como la eólica, solar, biomasa y geotérmica.

Este potencial, junto con la retracción de la demanda debido al estancamiento del crecimiento económico del país, ha generado que actualmente nuestro país tenga un excedente de producción de energía que se mantendría vigente por lo menos durante los próximos cinco o seis años. Este escenario nos lleva a pensar en opciones para ampliar la demanda de energía para los próximos años, siendo la interconexión eléctrica con los países vecinos en vías de una integración eléctrica regional, la mejor opción de incrementarla considerablemente a precios mayores que en el mercado interno, así como para brindarle mayor dinamismo a nuestro mercado eléctrico.

Sobre este tema, existe importante doctrina y análisis internacional, pero en el ámbito local, son pocos los trabajos que aportan a la discusión, siendo uno de los que más destaca el realizado por Margarett Matos Ortega en el 2016, a propósito de la publicación del Proyecto de Ley $\mathrm{N}^{\circ} 05201 / 2015-\mathrm{PE}^{1}$, en el cual encontramos una revisión extensiva del proyecto normativo $y$, a su vez, un desarrollo respecto a las posibilidades de exportación e importación de energía eléctrica en dicho contexto.

A la fecha, los numerosos acercamientos entre los Gobiernos de Chile y Perú han avivado nuevamente la discusión sobre las posibilidades de interconexión y de una futura integración eléctrica regional. Todas estas premisas han motivado el análisis que realizaremos a lo largo del presente artículo, en el cual consideraremos la experiencia internacional, la situación actual de nuestro país y, finalmente, presentaremos un conjunto de propuestas que engloban los aspectos que, a nuestro criterio, deberían tomarse en cuenta para que las interconexiones sean beneficiosas para los países involucrados, nos permitan exportar energía a precios competitivos o importarla cuando la requiramos y viabilizar en un futuro una integración eléctrica regional.

1. MATOS, Margarett. "Integración Eléctrica: ¿Perú, un país exportador e importador de energía eléctrica?" Consultado el 15 de septiembre de 2017. Disponible en: $<$ http://enfoquederecho.com/civil/integracion-electrica-peru-un-paisexportador-e-importador-de-energia-electrica/>. 


\section{MARCO TEÓRICO}

A fin de poder desarrollar qué implica para nuestro país el hecho de enrumbarnos en una integración eléctrica de tipo regional, vamos a identificar algunos conceptos importantes, con el objetivo que, durante el desarrollo del presente artículo, podamos no solamente entenderla, sino también poder analizar cuáles serían los beneficios y desventajas de su implementación, teniendo en cuenta la experiencia internacional.

\section{1. ¿Qué es la Integración Eléctrica?}

Al hablar de integración eléctrica, nos referimos al proceso en el cual un conjunto determinado de países decide, a través de un pacto expreso, interconectarse a través de sus sistemas eléctricos, con el objetivo de realizar transacciones internacionales de energía ${ }^{2}$. Estos intercambios pueden darse bajo diversas circunstancias, entre países con una matriz energética que requiere optimizarse o complementarse, con otros que tienen una matriz energética mucho más diversa, que les permite contar con producción de electricidad que puede ser utilizada para intercambios de energía.

Esta interconexión de sistemas eléctricos y los intercambios de electricidad, más allá de que necesiten, como aspecto físico, la infraestructura esencial para dicho propósito, requieren de un marco legal regulatorio, cuyo ámbito de aplicación alcance a todos los países involucrados en el pacto previo y donde se consignen los mecanismos, modalidades, restricciones y otras consideraciones que los países deben tener en cuenta, para viabilizar el proceso de importación o exportación de electricidad.

Bajo ese contexto, resulta importante entonces dar una revisión al panorama internacional, a efectos de analizar las experiencias en países pioneros en temas de integración eléctrica o energética en general, para así poder identificar cuáles han sido sus ventajas y/o desventajas en cuanto a su desarrollo e implementación:

\section{Experiencias internacionales de éxito en Integración Eléctrica.}

\subsection{Europa.}

Una de las experiencias más notables de integración eléctrica se ha dado en los mercados energéticos de la Unión Europea, que en últimas décadas viene trabajando intensamente para construir el mercado común más integrado, competitivo y sostenible de energía del mundo. Dicha integración está dando frutos tangibles, como por ejemplo que los precios de la electricidad al por mayor han disminuido en una tercera parte; los consumidores tienen más opciones donde elegir al paso que los proveedores de energía compiten para ofrecer mejores servicios con precios más bajos; y el marco jurídico ha mejorado la competitividad en el sector. Esta integración tiene como característica el haberse dado dentro del marco de integración económica de la Unión y no sólo como intercambios eventuales o de excedentes.

La integración energética, denominada Unión de la Energía, es necesaria en la UE pues es actualmente el mayor importador de energía del mundo, al importar el $53 \%$ de toda la energía que consume a un coste anual de unos 400000 millones de euros. Cabe señalar que esta integración se dio a través de tratados. A través del Tratado de Maastricht en 1993 y la creación de la Unión Europea, los países miembros se comprometieron a trabajar para armonizar los mercados energéticos locales con el fin de elaborar una política energética común. Este proceso no ha sido fácil, y sus principales dificultades han sido las siguientes:

a) las diferencias en la situación económica de inicio de los países miembros,

b) la diversidad de la estructura de suministro y consumo de energía,

2. Loc. Cit. 
c) la presencia de fuertes intereses de monopolios públicos, y

d) la reticencia de los Estados en dejar la política energética nacional en manos de una entidad supranacional ${ }^{3}$.

No obstante, la Comisión Europea, a través de un Estudio denominado"Paquete sobre la Unión de la Energía" de fecha 25 de febrero de 2015, ha concluido que la UE necesita elevar su nivel de interconexión de electricidad hasta el 10\% de aquí a 2020 en su empeño por crear una Unión de la Energía resiliente, con una política climática dotada de perspectiva de futuro ${ }^{4}$.

Otra de las experiencias internacionales de éxito en integración eléctrica en Europa es el Nordpool o Nordic Power Exchange, iniciado en 1996 por Noruega, después de haberse introducido en 1991 reformas en sector eléctrico que permitían la competencia. El Nordpool gestiona el mayor mercado de energía eléctrica de Europa y que opera en los países como Noruega, Estonia, Letonia, Suecia, Dinamarca, Finlandia, Lituania, Alemania y Reino Unido, comercializando más del $80 \%$ del consumo total de energía eléctrica de los países Nórdicos 5 .

El éxito del Nordpool se basa en la larga tradición de cooperación eléctrica entre los países escandinavos que permitían el libre acceso a los agentes a las redes de transmisión y propiciaban la competencia entre los generadores y es un gran ejemplo de que las reformas que introducen competencia pueden funcionar sin transferir la propiedad de las empresas eléctricas al sector privado. El Nordpool es una asociación voluntaria que existe en paralelo con los mercados domésticos y realiza sólo transacciones a nivel mayorista ${ }^{6}$.

\subsection{América del Norte.}

Otro caso importante es el de los países de América del Norte, donde los tres países que la constituyen, México, los Estados Unidos y Canadá, tienen en el Tratado de Libre Comercio de América del Norte o NAFTA, el marco legal para la creación de un mercado integrado de energía. Sin embargo, hasta hoy los alcances de la integración están limitados a acuerdos bilaterales, por un lado, entre Canadá y los EUA y, por otro, entre los EUA y México, principalmente por la gran asimetría del poder económico y político de los Estados Unidos?.

Si bien América del Norte posee el mercado de electricidad más activo e integrado del hemisferio, el comercio de electricidad entre los mismos está claramente diferenciado por el número de transacciones entre Canadá y Estados Unidos y el comercio entre México y los Estados Unidos. Las pequeñas transacciones entre México y los Estados Unidos son limitadas debido a la poca interconexión de las redes trasfronterizas, la armonización del mercado y a problemas de incompatibilidad de redes, hay una necesidad real de una red más extensa, lo cual requiere el desarrollo y actualización de la infraestructura actual para asegurar la confianza en el suministro ${ }^{8}$.

3. Apuntes sobre la Integración Eléctrica Regional y Propuestas para Avanzar. Organización Latinoamericana de Energía - OLADE. Consultado el 15 de septiembre de 2017. Disponible en: <http://www.olade.org/wpcontent/uploads/2015/08/INTEGRACI\%C3\%93N-EL\%C3\%89CTRICA-REGIONAL_Y_PROPUESTAS-PARAAVANZAR.pdf $>$.

4. Comisión Europea. Bruselas 25.02.2015 COM - 2015- 82 final. Paquete sobre la Unión de la Energía. Consultado el 15 de septiembre de 2017. Disponible en: <http://eur-lex.europa.eu/resource.html?uri=cellar:a5bfdc21bdd7-11e4-bbe1-01aa75ed71a1.0009.02/DOC 1\&format=PDF $>$.

5. Loc. Cit.

6. Loc. Cit.

7. Loc. Cit.

8. Cooperación e Integración Eléctrica Regional en las Américas. Organización de los Estados Americanos - OEA. Consultado el 15 de septiembre de 2017. Disponible en: <https://www.oas.org/dsd/Documents/ Cooperaci\%C3\%B3n\%20e\%20Integraci\%C3\%B3nEl\%C3\%A9ctricaRegionalenaAm\%C3\%A9ricas.pdf >. 


\subsection{América Latina.}

América Latina ya posee un fuerte grado de integración eléctrica. Desde el año 1995 hasta hoy, las interconexiones han aumentado aproximadamente diez veces, pasando de 500 MW a $5.000 \mathrm{MW}$ de capacidad instalada9. Estas integraciones se han dado de tres maneras. La primera de ellas es la construcción de plantas hidroeléctricas binacionales, tales como Salto Grande -Argentina y Uruguay, 1.800 MW-en 1979; Itaipú —Paraguay y Brasil, 14.000 MWen 1984; y Yacyretá —Paraguay y Argentina, $1.800 \mathrm{MW}$ en su primera fase- en $1998^{10}$.

Otro tipo de integración es la exportación de electricidad, como la que se viene danto entre Ecuador y Colombia, a través de una interconexión de 230 kV y $500 \mathrm{MW}$ de capacidad, que alcanza picos de hasta $7000 \mathrm{MWh}$ diarios ${ }^{11}$. No obstante, un caso interesante de exportación de electricidad es el de Uruguay, quien pasó de una crisis energética en el 2006 a causa de una sequía prolongada, a contar con excedentes y el desafío de comercializarlos, debido a un gran cambio en su matriz energética que resultó en la alta generación de energía eléctrica por fuentes renovables -eólica e hidroeléctrica básicamente- que excede la demanda local, lo que les ha permitido iniciar a exportar a Argentina y Brasil ${ }^{12}$.
En el caso de Uruguay y Brasil, la interconexión se dio a través de inversión binacional. Los proyectos se remontan al año 1997, cuando se firmó un memorándum de entendimiento para realizar acciones concretas de interconexión. A partir de aquel acuerdo, UTE de Uruguay y Eletrobras de Brasil encararon una primera inversión conjunta de US\$ 30 millones, para una planta conversora en la frontera entre Rivera y Livramento para interconectar y convertir hasta 70 megavatios, que entró en funcionamiento en $2001^{13}$.

En marzo de 2010 se firmaron los acuerdos entre UTE y Eletrobrás para comenzar con el segundo proyecto, a localizarse en la capital de Cerro Largo, de interconexión de redes de 500 $\mathrm{kV}$, que requirió la instalación de una estación conversora de frecuencia en las cercanías de la ciudad de Melo -Uruguay_-, de 500 MW de potencia, debido a que los sistemas de ambos países operan en diferente frecuencia -50 y 60 $\mathrm{Hz}$ respectivamente $-{ }^{14}$.

Finalmente, dentro de América Central también se ha dado otro tipo de integración eléctrica, con proyectos donde se comparten las reservas y se aprovecha la diversidad hidrológica ${ }^{15}$. Este proceso ha sido promovido por varias décadas como un instrumento fundamental para el desarrollo económico y la integración regional.

9. MIELGO, Pedro. En: Banco de Desarrollo de América Latina: "Nuevas oportunidades de Interconexión Eléctrica en América Latina", p. 20. Consultado el 16 de septiembre de 2017. Disponible en: <http://publicaciones.caf.com/ media/18406/oportunidades-interconexion-electrica-america-latina.pdf $>$.

10. Loc. Cit.

11. "Ecuador exportará energía a Colombia". En: CELEC. Ecuador: 2017. Consultado el 17 de septiembre de 2017. Disponible en: <https://www.celec.gob.ec/78-quienes-somos/310-ecuador-exportara-energia-acolombia.html>.

12. "UTE exporta electricidad a la vez a Brasil y Argentina". En: El País Uruguay. Consultado el 21 de septiembre de 2017. Disponible en: <https://negocios.elpais.com.uy/noticias/ute-exporta_electricidad-vez_brasil_argentina. $\underline{\text { html }}>$.

13. "Planta conversora de UTE en Cerro Largo está lista, pero su uso aparece incierto a corto plazo debido a la caída de la demanda energética en Brasil". En: Perspectiva. Consultado el 21 de septiembre de 2017. Disponible en: < $\underline{\text { https:// }}$ www.enperspectiva.net/enperspectiva - net/informes/informe-planta-conversora-de-ute-en-cerrolargo-esta-en-condiciones-de-operar-pero-caida-de-demanda-energetica-brasilenagenera-incertidumbre/>.

14. Ficha de proyecto: Interconexión eléctrica entre Uruguay y Brasil. Consultado el 21 de septiembre de 2017. Disponible en: $<$ http://www.iirsa.org/proyectos/detalle proyecto.aspx?h=1278>.

15. Banco de Desarrollo de América Latina. Op.cit. p. 20. 
Hasta el día de hoy se han logrado enormes avances y resultados gracias a la integración energética, gracias al compromiso político que se plasmó en 3 convenios internacionales: el Tratado Marco y el Primer y Segundo Protocolo de dicho tratado ${ }^{16}$.

La firma de estos convenios y los esfuerzos por avanzar en la integración eléctrica regional se han materializado en el diseño y ejecución del proyecto "Sistema de Interconexión Eléctrica de los Países de América Central" — SIEPACque contiene la infraestructura de interconexión regional compuesta por la línea SIEPAC, que se extiende de Guatemala a Panamá a lo largo de 1,800 Km de longitud. Esta línea de transmisión fue diseñada para intercambiar 300MW de electricidad ${ }^{17}$.

Para la puesta en marcha de un proyecto emblemático como es el Mercado Eléctrico Regional - MER - , se requirió el desarrollo de la arquitectura institucional, normativa y regulatoria que permitió su pleno funcionamiento $y$ operación en la actualidad. La puesta en marcha de la infraestructura y el mercado posibilitó que los intercambios entre los agentes en el MER se incrementaran un $580 \%$ en los últimos 5 años, y aumentara también la cantidad de empresas generadoras, comercializadoras, distribuidoras y grandes usuarios - que en la actualidad alcanzan a 240 agentes - que participan en el MER ${ }^{18}$.

\section{Ventajas y desventajas en la experiencia internacional.}

Luego de la experiencia internacional antes descrita, vemos que una de las ventajas principales que trae la integración eléctrica, es la posibilidad de reducir situaciones de escasez de energía periódicas en la región correspondiente, contribuyéndose a la seguridad del suministro entre los países interconectados, facilitando funciones de apoyo entre sistemas vecinos y aportando mayor estabilidad y garantía de la frecuencia en los sistemas interconectados. Esta ventaja va de la mano con la oportunidad de la comercialización de los excedentes de energía, que si bien suponen inversiones fuertes por parte de los países involucrados, estas se justifican y compensan con los ingresos económicos obtenidos por la exportación.

Asimismo, al optimizar el uso compartido de la energía se reducen los costos de operación, fomentándose un mejor aprovechamiento de los recursos energéticos. De igual manera, al facilitar los intercambios comerciales de energía, se aprovechan las diferencias de precios de la energía en los sistemas eléctricos interconectados, aumenta la competencia y al tener un mercado energético competitivo esto puede atraer la inversión extranjera en nuevos proyectos.

No obstante, podemos apreciar que los procesos de integración eléctrica casi siempre han tomado un carácter político, lo que usualmente ha generado obstrucciones en la organización e integración de las voluntades e intereses de los países involucrados. Asimismo, en algunos casos la asimetría económica entre los países involucrados ha generado obstáculos al desarrollo de estos acuerdos.

En adición a ello, existe usualmente incertidumbre inicial respecto al costo por la infraestructura de interconexión eléctrica necesario para la integración y las vías por las cuales puede hacerse

16. Integración Eléctrica Centroamericana. Génesis, Beneficios y Prospectiva del Proyecto SIEPAC —Sistema de Interconexión Eléctrica de los Países de America Central-.p. 2. Consultado el 16 de septiembre de 2017. Disponible en: $\quad<$ https://publications.iadb.org/bitstream/handle/11319/8237/Integracion-electrica-centroamericanaGenesis-beneficios-y-prospectiva-del-Proyecto-SIEPAC-Sistema-de-Interconexion-Electricade-los-Paises-de-America-Central.PDF>.

17. Loc. Cit.

18. ECHEVARRÍA, Carlos. Desafíos y avances al desarrollo del Mercado Eléctrico Regional -MER-. Consultado el 16 de septiembre de 2017. Disponible en: $<$ https://blogs.iadb.org/energia es/2017/08/08/desafios_y—avances—al_ desarrollo_del_mercado-electrico-regional-mer/>. 
viable teniendo en cuenta las diferencias en la situación económica de inicio de los países involucrados, así como la diversidad de la estructura de suministro y consumo de energía.

\section{PANORAMA ACTUAL EN EL PERÚ}

De acuerdo con el COES, las interconexiones internacionales del Perú con países vecinos tienen un alto potencial de desarrollo hacia el mediano y largo plazo, pues indican que hasta el año 2026 el SEIN contará con un sistema de transmisión de $500 \mathrm{kV}$ con alcance geográfico cercano a las fronteras de varios de los países vecinos y con oferta de generación suficiente que permitirá tener capacidad de intercambio de electricidad con esos países conforme a los acuerdos binacionales o regionales a los que se arribe ${ }^{19}$.

En la actualidad, si bien el Perú no cuenta con ninguna interconexión eléctrica permanente, sí mantiene suscritos acuerdos comerciales de integración eléctrica con Brasil y Ecuador. Asimismo, existe el esfuerzo de llegar a un acuerdo binacional que permita viabilizar la interconexión eléctrica con Chile, hecho que viene generando discusión en nuestro país.

A continuación, vamos a abarcar cada caso con mayor detalle:

\section{Interconexión Perú - Ecuador.}

La interconexión eléctrica entre el Perú y Ecuador es en la que más se ha avanzado. Desde el 2004, contamos con un enlace de 220 kV de capacidad limitada - $160 \mathrm{MW}-$, que une a las Sub Estaciones de Zorritos - Perú-Machala-Ecuador-. La implementación de éste se dio luego de un primer estudio en el año 2001 y en el marco de la Decisión 536 de la CAN, que brindó el marco jurídico comunitario para impulsar el desarrollo del tema eléctrico entre los Países Miembros ${ }^{20}$.

Para regular las transacciones de importación y exportación de electricidad en los aspectos operativos, comerciales y tarifarios, y prever la operación no sincronizada del enlace entre Perú y Ecuador, se publicó en diciembre de 2004 el Decreto Supremo № 045-2004-EM, mediante el cual fue aprobado el Reglamento de Importación y Exportación de Electricidad —en adelante, "RIEE" - , sustituido por el Decreto Supremo No 049-2005-EM.

No obstante, fue recién en el año 2012 que se viabilizaron los intercambios de energía a través de normativa en ambos países. En el Perú, con la derogación de la Decisión 536 y su reemplazo con la Decisión 757 publicada el 22 de agosto de 2011, que incorporó en su Anexo 2 un régimen temporal para los intercambios eléctricos entre Ecuador y Perú, se aprobó en mayo de 2012 un Reglamento Interno para la aplicación de esta Decisión, el Decreto Supremo N ${ }^{\circ} 011$ 2012-EM y el Procedimiento Técnico COES $N^{\circ}$ 43"Intercambios Internacionales de Electricidad en el marco de la Decisión 757 de la CAN". En Ecuador, se emitió el Decreto Ejecutivo 3448 y la Regulación CONELEC 002/12 “Transacciones Internacionales de Electricidad entre Ecuadory Perú aplicables durante el período transitorio acorde con lo dispuesto en la Decisión 757 de la Comunidad Andina"21.

Finalmente, en julio de 2012 los operadores de los sistemas eléctricos de ambos países —el

19. COES Informe COES/D/DP-01-2016 "Propuesta de Definitiva de Actualización del Plan de Transmisión 20172026". Consultado el 15 de septiembre de 2017. Disponible en: <http://www.coes.org.pe/portal/browser/ download?url=Planificaci\%C3\%B3n\%2FPlan\%20de\%20Transmision\%2FActualizacion\%20Plan\%20de\%20 Transmision\%202017\%20-\%202026\%2F09 PropuestaDefinitiva\%2FVolumen\%20I Informe\%2F09.\%20 Interconexiones\%20Internacionales.pdf>.

20. Estudio Interconexión Perú - Ecuador, desarrollado por Hydro-Québec Intl., para ETECEN (Perú) y TRANSELEC (Ecuador), citado en COES Informe COES/D/DP-01-2016. Op. Cit.

21. MATOS, Margarett. Op. cit. 
COES en Perú y el CENACE en Ecuador-celebraron un Acuerdo Operativo para coordinar la operación de las transacciones de electricidad a corto plazo. Es importante indicar que, si bien a través del marco normativo descrito se vienen realizando intercambios de importación y exportación de energía con el enlace actual, en la práctica éste sólo ha operado en situaciones extraordinarias y generalmente de emergencia, para evitar la interrupción del suministro eléctrico en ambos países ${ }^{22}$.

Por ello, para posibilitar la interconexión permanente entre ambos países y fomentar un mejor aprovechamiento de los recursos energéticos disponibles, en noviembre de 2013 se suscribió el Acuerdo de Piura, por el que se acordó dar inicio al proyecto para la construcción y puesta en marcha de una línea de interconexión eléctrica de $500 \mathrm{kV}$ para ambos países, la Línea de Transmisión SE La Niña —Perú-, SE Chorrillos -Ecuador- de $587 \mathrm{~km}$, que se encuentra prevista en el Plan de Transmisión actual ${ }^{23}$. Cabe indicar que los estudios para la viabilidad de esta línea se realizaron en el marco del Sistema de Interconexión Eléctrica Andina -en adelante, "SINEA" - ${ }^{24}$ y fueron realizados por el Banco Interamericano de Desarrollo -en adelante, "BID" - en el año 2014, el mismo que tuvo como objetivo seleccionar las alternativas de interconexión más convenientes desde un punto de vista técnico, económico, regulatorio, político, ambiental y social entre los países miembros.

\section{Interconexión Perú - Brasil.}

La interconexión con Brasil tuvo incentivos prometedores, pero actualmente los planes para la misma se encuentran prácticamente en pausa. En mayo de 2008, se suscribió el Convenio de Integración Energética Perú-Brasil, que tuvo por objetivos principales el desarrollar estudios sobre el potencial energético de ambos países, evaluar proyectos hidroeléctricos para la exportación de energía eléctrica al Brasil, evaluar marcos normativos, analizar proyectos de conexión fronteriza e intercambiar experiencia en diversos aspectos energéticos ${ }^{25}$. Luego de este proceso, se identificó un potencial hidroeléctrico de $20000 \mathrm{MW}$ ubicado en la vertiente oriental de la cordillera de los Andes ${ }^{26}$.

En el año 2010, hubo un intento fallido de pactar la exportación de excedentes de electricidad al Brasil, a través de la suscripción del Acuerdo Energético Perú-Brasil. Dicho acuerdo fomentaba la construcción de cinco proyectos de plantas con capacidades instaladas entre 800 y 2200 MW, de los cuales el proyecto de la Central de Inambari -2 $200 \mathrm{MW}$ - fue el que más interés despertó por parte de Brasil $^{27}$. Pese a ello, el proyecto Inambari, valorizado en US\$ 4,000 millones, fue paralizado en el año 2011 debido a irregularidades en su Estudio de Impacto Ambiental y a las protestas de la población afectada, porque implicaba un desplazamiento forzoso e incluso daños ambientales ${ }^{28}$.

22. Loc. Cit.

23. COES Informe COES/D/DP-01-2016. Op. Cit.

24. El SINEA es la iniciativa de integración eléctrica regional impulsada en el marco de la Comunidad Andina, que comprende a Colombia, Ecuador, Perú, Bolivia —en calidad de observador — y Chile — miembro asociado—.

25. COES Informe COES/D/DP-01-2016. Op. Cit.

26. Banco de Desarrollo de América Latina. Op.Cit. p. 35.

27. Loc. Cit.

28. Comisión de Relaciones Exteriores archivó dictamen que buscaba aprobar Acuerdo Energético Perú - Brasil. SPDA Actualidad Ambiental. Consultado el 16 de septiembre de 2017. Disponible en: <http://www.actualidadambiental. $\mathrm{pe} / \mathrm{p}=23142>$

29. Dictamen recaído en el Proyecto de Resolución Legislativa N $N^{0} 391 / 2011$-PE. Consultado el 15 de septiembre de 2017. Disponible en: <http://www.actualidadambiental.pe/wp_content/uploads/2014/05/Dictamen—dleAcuerdo-Energ\%C3\%A9tico-Per\%C3\%BA-Brasil.pdf $>$. 
Finalmente, el Acuerdo Perú-Brasil no fue aprobado por el Congreso peruano y fue archivado en mayo de $2014^{29}$. El Congreso consideró que se planteaba exportar energía al Brasil como obligación y no como decisión de los agentes en base a los incentivos del mercado, comprometiendo la nueva generación eléctrica del Perú con la exportación al Brasil de manera exclusiva por al menos 30 años, además de costos importantes y potenciales de carácter ambiental ${ }^{30}$.

\section{Interconexión Perú - Chile.}

Pese a que hasta el momento no se cuenta con un acuerdo binacional, la interconexión con Chile podría ser una realidad en el corto plazo, por los diversos avances que se han dado en los últimos años. Si bien la situación energética de Chile ya no se encuentra en la crisis de hace algunos años y vienen impulsando una generación eléctrica importante a través de Recursos Energéticos Renovables - RER - en su mayoría mediante centrales solares, su generación RER requiere un respaldo debido al bajo factor de plata que este tipo de tecnología suele tener ${ }^{31}$. Aquí hay un campo importante que nuestro país puede aprovechar para exportación de energía.

Ahora bien, en el marco de los estudios descritos líneas atrás realizados por el SINEA, en el caso de Chile se plantearon dos alternativas viables: una línea de 220 kV desde Tacna a Arica — Los Héroes - Arica, 70 km-y una línea en corriente continua de $500 \mathrm{kV}$ desde Moquegua al centro de Chile -Montalvo - Crucero, $650 \mathrm{~km}$ - ${ }^{32}$.

Sobre los proyectos mencionados, en el último año el COES -Perú- y el Centro de Despacho de Carga del Sistema Norte Grande de Chile -CDEC-SING - Ilevaron a cabo un estudio que tuvo como objetivo desarrollar los análisis, a nivel de factibilidad y elaborar la ingeniería a nivel de licitación de concesión de la línea de 220 kV33.

Asimismo, en junio de 2017 los Ministros de Energía y Minas de Perú y Chile suscribieron los términos de referencia para la elaboración del estudio de interconexión eléctrica entre ambos países, que contempla la construcción de la referida línea de transmisión de 220 kV$^{34}$. Este estudio ya fue encargado al Banco Interamericano de Desarrollo - BID—, según lo suscrito en julio de este año en la Declaración de Lima en el marco del Gabinete Binacional Perú-Chile; se estima que el proceso de interconexión podría tomar entre 48 a 72 meses, pudiendo concretarse entre el 2019 y $2020^{35}$.

Finalmente, en el marco del evento "Día de la Energía" llevado a cabo en octubre de 2017, los Ministros de Energía y Minas de Perú y Chile dieron cuenta de los avances del proceso de interconexión, indicando que en dicho mes los organismos reguladores se estarían reuniendo para ultimar los detalles de los aspectos regu-

30. MATOS, Margarett. Op cit.

31. El factor de planta - también llamado factor de capacidad neto o factor de carga- de una central eléctrica es el cociente entre la energía real generada por la central eléctrica durante un período - generalmente de forma anual - y la energía generada si hubiera trabajado a plena carga durante ese mismo período. Es una indicación de la utilización de la capacidad de la planta en el tiempo. Las centrales solares tienen un bajo factor de planta debido a que dependen del recurso solar, que sólo está disponible una parte del día.

32. COES Informe COES/D/DP—01-2016. Op. Cit.

33. Loc. Cit

34. "Se firma acuerdo para elaborar estudio de interconexión eléctrica entre el Perú y Chile". En: Diario El Comercio. Consultado el 11 de julio de 2017. Disponible en: <http://elcomercio.pe/economia/firma-acuerdo-elaborarestudio-interconexion-electrica-peru-chile-436802>.

35. "MEM: Interconexión eléctrica entre Chile y Perú se hará realidad entre el 2019 y 2020". En: Diario Gestión. Consultado el 20 de septiembre de 2017. Disponible en: <http://gestion.pe/economia/mem—interconexion—electricaentre-chile-y-peru-se-hara-realidad-entre-2019-y-2020-2194992>. 
latorios necesarios para que esta interconexión sea beneficiosa para ambos países.

\section{Interconexión Perú - Bolivia.}

Si bien Bolivia es parte del SINEA, no contamos aún con un acuerdo binacional ni estudios específicos de interconexión eléctrica, pero a diferencia del caso de Chile, son pocos los avances que se han hecho al respecto. La razón para ello es que, de acuerdo con el COES y a los estudios encargados por el SINEA, una interconexión entre Perú y Bolivia tendría un mayor costo respecto a un enlace sincrónico convencional, debido a la diferencia de frecuencia entre ambos sistemas —Perú: $60 \mathrm{~Hz}$ y Bolivia: $50 \mathrm{~Hz}$-, lo que obligaría al uso de convertidores o un enlace en corriente continua ${ }^{36}$.

Otro factor que desincentiva la interconexión entre Perú y Bolivia es la baja complementariedad estacional entre ambos sistemas, pues en lo que se refiere al desarrollo de la generación, por el lado peruano se tiene un sistema de generación hidro-térmica, mientras que en el lado boliviano predomina la generación a gas natural, razón por la cual se espera que las diferencias de costos marginales entre ambos sistemas sean relativamente pequeñas, debido a que los costos marginales en ambos países están definidos por la generación a gas natural local ${ }^{37}$.

En cuanto a acuerdos entre ambos países, en junio de 2015 los Ministros de Energía y Minas de Perú y Bolivia firmaron un Acta para la conformación de un Comité Binacionales de Hidrocarburos y de Energía Eléctrica, que abordaría temas de interconexión eléctrica y un futuro convenio bilateral de integración eléctrica ${ }^{38}$.

\section{Consideraciones sobre la integración eléctrica regional.}

Todos los casos detallados constituyen los esfuerzos que se vienen realizando para una futura integración eléctrica regional. La Comunidad Andina de Naciones - CAN-, a través de su Agenda Estratégica Andina - AEA - ha identificado a la integración energética como una de las doce áreas estratégicas relevantes. En atención a ello, ha venido aprobando decisiones con el fin de impulsar la normativa en temas de interconexión de los sistemas de electricidad de los Países Miembros - Bolivia, Colombia, Ecuador y Perú-.

En diciembre de 2002, se aprobó la Decisión 536 "Marco General para la Interconexión Subregional de Sistemas Eléctricos e Intercambio Intracomunitario de Electricidad"39, que brindó el marco jurídico comunitario para impulsar el desarrollo del tema eléctrico entre los Países Miembros. Esta Decisión fue posteriormente suspendida por la Decisión 720 —noviembre de 2009-y reemplazada por un "Régimen Transitorio Aplicable a las Transacciones Internacionales de Electricidad entre Ecuador y Perú" mediante la Decisión $757^{40}$, régimen vigente para el periodo de agosto de 2011 a agosto de 2016.

Cabe señalar que estos esquemas fueron transitorios y que en abril de este año se aprobó finalmente la Decisión 816, "Marco General para la Interconexión Subregional de Sistemas Eléctricos e Intercambio Intracomunitario de

36. COES Informe COES/D/DP-01-2016. Op. Cit.

37. Loc. Cit.

38. "Perú y Bolivia conforman Comité Binacional de Hidrocarburos y Energía". Andina Perú. Consultado el 20 de septiembre de 2017. Disponible en: <http://www.andina.com.pe/agencia/noticia-peru-y—boliviaconforman-comite-binacional-hidrocarburos-y-energia-electrica-558982.aspx>.

39. CAN, 2002. Decisión 536. Consultado el 21 de septiembre de 2017. Disponible en: <http://intranet. comunidadandina.org/Documentos/decisiones/DEC536.doc $>$.

40. CAN, 2011. Decisión 757. Consultado el 21 de septiembre de 2017. Disponible en: <http://intranet. comunidadandina.org/Documentos/decisiones/DEC757.doc >. 
Electricidad" que prevé el establecimiento del Mercado Andino Eléctrico Regional -en adelante, "MAER"-. El objetivo de este marco regulatorio y el MAER propiamente es que funcionen como un mercado de excedentes eléctricos en el que no se afectará el suministro interno de los Países Miembros. Por el contrario, el MAER permitirá que, en casos de emergencia y desabastecimiento interno, se pueda suplir la demanda interna y así evitar racionamientos eléctricos ${ }^{41}$.

Esta decisión por parte de la Comunidad Andina tiene correlación con el Proyecto de Ley $\mathrm{N}^{\circ}$ 05201/2015-PE presentado por el Ejecutivo en abril de 2016: "Ley que aprueba el marco general para la interconexión internacional de los sistemas eléctricos y el intercambio de electricidad"42. Este proyecto de ley plantea los siguientes lineamientos generales:

a) Tanto la interconexión de sistemas eléctricos y los intercambios de electricidad se realizarán siempre que previamente se haya suscrito un acuerdo internacional específico ente los países involucrados.

b) El Perú solo podrá exportar sus excedentes de producción de electricidad, teniendo siempre prioridad la demanda interna. En situaciones de emergencia, el COES podrá disponer la interrupción de la exportación.

c) Las exportaciones no deben generar incrementos en el Mercado Mayorista de Electricidad ni sobrecostos para los usuarios eléctricos peruanos.

d) Los intercambios estarán determinados y programados por decisiones operativas de despacho económico coordinado entre el COES y los operadores de los otros siste- mas, no debiendo generar restricciones ni congestión de líneas de transmisión para el mercado interno.

e) Las transacciones por intercambios de electricidad no pueden considerar en ningún caso al SEIN como sistema de tránsito, no es posible otorgar concesiones de transmisión que atraviesen nuestro territorio, sin conectarse al sistema eléctrico peruano.

f) Se establece que los acuerdos internacionales fijarán los precios para la exportación de electricidad, considerando los costos marginales e el nodo de frontera, costos adicionales para el mercado interno imputables a los intercambios, así como un mecanismo de compensación a favor del Estado peruano por el intercambio de electricidad generada con recursos naturales, que considere el precio doméstico y el precio comparable del gas natural de destino, utilizado para la generación eléctrica.

g) Las transacciones internacionales de electricidad serán de corto plazo, el sistema eléctrico con el precio más económico en el nodo de frontera será el que exporte energía al otro sistema.

h) El pago de la exportación de electricidad debe estar respaldado por una garantía de parte de los importadores de electricidad.

i) Se crea el mecanismo de la Compensación Tarifaria con el objeto de reducir los cargos adicionales en el peaje de transmisión, y así trasladar el beneficio económico obtenido en los intercambios a los usuarios finales. Esta compensación estaría destinada a financiar algunos de los cargos adicionales que actualmente no permiten que las

41. CAN, 2017. Decisión 816. Consultado el 21 de septiembre de 2017. Disponible en: <http://intranet. comunidadandina.org/Documentos/decisiones/DEC816.docx $>$.

42. Proyecto de Ley $\mathrm{N}^{\circ}$ 0521/2015-PE. Congreso de la República. Consultado el 22 de septiembre de 2017. Disponible en: <http://www2.congreso.gob.pe/Sicr/TraDocEstProc/Contdoc03 2011.nsf/ dff31fef3f6852be05257e22000b22a3/8b1d02680b88645705257f8800587466/\$FILE/PL0520120160401.pdf>. 
tarifas de electricidad se reduzcan ${ }^{43}$, así como en la inversión en la infraestructura necesaria para la interconexión ${ }^{44}$.

Como vemos, la política expresada en este Proyecto de Ley propone que el beneficio comercial de las exportaciones se dirija a la reducción de las tarifas de los usuarios del servicio regulado de electricidad, quienes a causa de los costos adicionales que se incluyen en la tarifa, hasta el momento no pueden verse beneficiados por los bajos costos de energía que ha generado la situación actual de sobreoferta de electricidad.

Si bien este proyecto de Ley fue debatido en mayo de 2016, lamentablemente hasta el momento no ha sido aprobado. Consideramos que esta iniciativa del Ejecutivo impulsa las posibilidades de nuestro país de convertirse en hub energético hacia el 2021 y poder exportar los excedentes de energía a Ecuador, Chile, Brasil y Bolivia ${ }^{45}$, pues abarca muchos de los aspectos que preocupan al Estado respecto a los intercambios de energía ${ }^{46}$.

Debemos considerar que si las interconexiones eléctricas internacionales se han extendido globalmente es precisamente por implicar diversas ventajas a los países involucrados, tanto a corto, mediano y largo plazo. El hecho de embarcarnos en una integración eléctrica de índole regional contribuye a la seguridad del suministro energético de los países que participen en ella, pues nos da la posibilidad de constituir una red donde un país pueda valerse del suministro eléctrico disponible en otro en situaciones de emergencia, a través de fuentes de energía más diversificadas. Asimismo, se abre la posibilidad de beneficiarnos de las diferencias de demanda tanto estacionales como diarias, así como de lograr menores costos de operación al optimizar el uso de recursos compartidos. Igualmente, un mercado de electricidad integrado regional mejoraría las condiciones de competencia, pues se incrementaría el número de competidores, limitando que se ejerza un poder de mercado por parte de unos pocos, reduciéndose así el riesgo de prácticas anticompetitivas, lo que fomentaría que la generación de energía se realice de manera cada vez más eficiente ${ }^{47}$.

No obstante, sin la aprobación de este proyecto y sin planes de inversión u otros incentivos similares por parte del Estado, el Perú no tiene establecida una política para el desarrollo de las interconexiones eléctricas internacionales, ni para la regulación de las transacciones eléctricas internacionales. El problema con esto es que el potencial energético de nuestro país y la sostenibilidad del suministro eléctrico en el futuro nos exige acelerar los procesos de exportación e importación de electricidad entre los países vecinos.

43. Actualmente, un porcentaje de las tarifas eléctricas corresponde a estos cargos adicionales, que se fijaron con la finalidad de promover la producción de energía, tales como la compensación a las centrales de generación con Recursos Energéticos Renovables — RER—, compensación por FISE, compensación a centrales de Reserva Fría, entre otros.

44. "Perú exportará electricidad a Chile y países vecinos". En: Mining Press. Consultado el 23 de septiembre de 2017. Disponible en: <http://www.miningpress.com/nota/294839/peru-busca-convertirse-en-hubenergetico-excedentes- $y$-tarifas $>$.

45. "Perú apunta a convertirse en hub energético exportando electricidad a Ecuadory Chile". En:Diario Gestion. Consultado el 22 de septiembre de 2017. Disponible en: <http://gestion.pe/economia/peru—apunta-convertirse-hubenergetico-exportando-luz-hacia-ecuador-y-luego-chile-2155715>.

46. "Las preocupaciones que tiene Perú sobre la integración eléctrica regional". En: Diario Gestión. Consultado el 22 de septiembre de 2017. Disponible en: <http://gestion.pe/economia/preocupaciones-que-tiene-perusobre-integracion-electrica-regional-2171716>.

47. VEIT, Böckers, HAUCAP, Justus, and HEIMESHOFF, Ulrich. "Benefits of an integrated European electricity market". Consultado el 22 de septiembre de 2017. Disponible en: < http://publications.europa.eu/resource/ cellar/99d4fd94-7619-44f4-9f4b-5541235b90d1.0001.04/DOC 1>. 


\section{CONCLUSIONES Y PROPUESTAS}

De los casos descritos tanto en el ámbito internacional y los avances regionales, es claro que no existe un patrón o un orden establecido de las cosas en los procesos de integración energética. No obstante, en el caso del Perú, creemos que los acuerdos bilaterales entre Estados con la finalidad de regular intercambios físicos de electricidad decididos por los agentes son un primer paso hacia una integración regional que beneficie a todos. Una vez que tengamos esta fase desarrollada, podemos avanzar hacia transacciones de corto plazo decididas por los operadores nacionales o multinacionales, por comparación de costos marginales.

Al hablar de interconexiones y de una integración eléctrica regional, debemos pensar en los beneficios para todos los países a largo plazo, no sólo en aspectos económicos sino también de seguridad energética. Por ello, la sobreoferta de electricidad que actualmente vive nuestro país no debe ser la única motivación para impulsar las interconexiones, lo que verdaderamente nos debe impulsar es lo que puede ocurrir cuando esta situación se revierta.

La interconexión Perú - Chile refleja justamente eso, la misma que ha avanzado considerablemente en los últimos años gracias a la voluntad de ambos Estados. Gracias a ello, ya se encuentran en desarrollo los estudios económicos respecto a la infraestructura necesaria para el proceso, así como algunas proyecciones respecto de proyectos a futuro. Consideramos que la adecuación de los marcos reguladores es un punto clave en el proceso, lo cual no debería ser un gran obstáculo al tener ambos países regulaciones bastante similares en temas energéticos. No obstante, aspectos como la remuneración y precios de los intercambios, costos por la infraestructura, posibles inversiones en tecnología y el modelo regulatorio propiamente para este negocio aún no han sido resueltos y requieren la mayor atención por parte de las entidades involucradas. De concretarse en el corto/mediano plazo, la interconexión con Chile marcaría un precedente importantísimo para el resto de posibles interconexiones y la integración eléctrica regional.

Por ello, necesitamos asegurar que estas interconexiones sean beneficiosas para los países y en nuestro caso, que nos permitan exportar energía a precios competitivos o importarla cuando la requiramos. Para ello, hemos resumido algunos aspectos a considerar:

a) La determinación de los precios de exportación e importación de energía debe ser recíproca y a precios de mercado, a fin de que los países involucrados no se vean perjudicados por subsidios o políticas internas de cada país.

b) Una adecuada capacidad de transmisión es esencial para los intercambios de energía, razón por la cual es importante definir el financiamiento de las líneas de transmisión que serán necesarias, ya sea a través de acuerdos bilaterales entre países donde se compartan costos o a través de empresas privadas.

c) Limitar en un principio los intercambios de electricidad a intercambios físicos de electricidad decididos por los agentes, que pueden ser excedentes de energía determinados por cada país. Una vez que se desarrolle este mercado regional, será posible aprovechar en mayor medida el potencial energético de los países que lo integren, a través de sus propios operadores y un operador regional.

d) El suministro interno de cada país debe tener siempre prioridad, razón por la cual debe especificarse que los intercambios de energía son interrumpibles, cuando pongan en riesgo la seguridad y confiabilidad del suministro interno.

e) Las exportaciones de electricidad no deben ocasionar incrementos considerables en la valorización de las transacciones del Mercado Mayorista de Electricidad fijados por la entidad competente para el mercado interno, ni sobrecostos a ser asumidos por los usuarios.

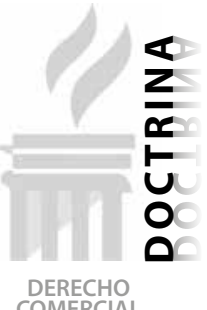


f) Las transacciones internacionales de electricidad con los países vecinos involucrados, dentro de su regulación, deberán estar previamente programadas y no deberán ocasionar restricciones 0 complicaciones para para la atención del mercado interno. Para ello, en un futuro es posible plantear la posibilidad de un órgano supranacional que se encargue de la programación de estos despachos.
Si bien a lo largo del presente artículo hemos detallado los avances de este proceso hasta el momento, es claro que aun existe mucho trabajo por hacer para concretar las interconexiones entre países vecinos y poder llegar una integración eléctrica regional que sea beneficiosa para todos los países que la integren. No obstante, vamos en buen camino y queda en manos de las entidades encargadas el llegar, con el debido análisis, a los acuerdos necesarios para ello. 